\title{
The Comparative Effect of Combination of Metformin and Megestrol Acetate With Megestrol Acetate Alone on Endometrial Growth Disorders
}

\author{
Manizheh Sayyah-Melli ${ }^{1 *}$, Sanam Pourazad ${ }^{1}$, Parvin Mostafa Gharebaghi ${ }^{1}$, Elaheh Ouladsahebmadarek ${ }^{1}$, \\ Mehri Jafari-Shobeiri ${ }^{1}$, Vahideh Rahmani ${ }^{1}$
}

\begin{abstract}
Objectives: Endometrial hyperplasia has an affinity for progression toward endometrial carcinoma. This study was conducted to compare the effect of metformin plus megestrol acetate with megestrol acetate alone on endometrial histology in the patients with endometrial proliferative and hyperplastic disorders.

Materials and Methods: In a single-blind clinical trial, 96 patients with abnormal uterine bleeding and endometrium with irregular or hyperplastic glands with or without atypical foundations were divided randomly into 2 equal groups. Group1 received metformin $500 \mathrm{mg}$ twice a day plus megestrol acetate $40 \mathrm{mg}$ daily for 3 months and group 2 received megestrol acetate $40 \mathrm{mg} / \mathrm{d}$ for $3 \mathrm{months}$. The treatment response was evaluated using pathologic samples of endometrial biopsies 2 weeks after completing the treatment. Results: In both groups, endometrial hyperplasia was resolved in most cases. Significant decrease was observed within groups in the endometrial thickness before and after the intervention. Comparison of thickness between 2 groups showed that in the megestrol acetate group, after intervention, significant decrease in the endometrial thickness was revealed (12.73 $\pm 6.04 \mathrm{~mm}$ [group 1], versus $8.55 \pm 3.43 \mathrm{~mm}$ [group 2], $P<0.001$ ). Lack of response to treatment was seen in $7 \%$ of group 1 and $4.9 \%$ of group 2 ( $P=0.68)$. None of the groups showed any complications and there was no hysterectomy.

Conclusions: Both treatment modalities were effective in treating proliferative and hyperplastic disorders. However, the response to treatment in the megestrol group was greater than that in the megestrol plus metformin group.

Keywords: Endometrial hyperplasia, Endometrial proliferative disorders, Metformin, Megestrol acetate
\end{abstract}

\section{Introduction}

Endometrial cancer is the most common cancer of the female genital tract and is the fourth most common cancer in women (1). Its prevalence increases with age and usually is diagnosed at the ages 45 to 65 (2).

Endometrial hyperplasia, especially atypical forms, has strong affinity for progression toward endometrial carcinoma. Hysterectomy is the preferred treatment for these patients. However, conservative treatment was reserved for the patients with severe medical problems that would make them very poor surgical candidates or for younger patients who would like to preserve their childbearing potential $(3,4)$. Progestins such as megestrol acetate and medroxy progesterone acetate are the most commonly used treatments. However, the therapeutic response, especially for the atypical type, is not always satisfactory. Oral progestins are accompanied by low compliance and systemic side effects that could limit their effectiveness (5). It was found that endometrial hyperplasia has obvious relationship with metabolic disorders and insulin resistance (6). Furthermore, some case reports have shown that patients with atypical endometrial hyperplasia who did not respond to progestin therapy could achieve complete improvement by adding metformin (7-9). Metformin is an insulin sensitizing agent and acts as an inhibitory factor against endometrial cancer by inhibiting the proliferation of malignant cells (10). Metformin with megestrol acetate has recently been identified as an alternative to the treatment of endometrial hyperplasia. However, clinical evidence is not available significantly (11). Further studies are needed to achieve therapeutic outcomes.

The present study aimed to compare the effect of metformin plus megestrol acetate with megestrol acetate in the treatment of endometrial proliferative and hyperplastic disorders.

\section{Materials and Methods}

This single-blind clinical trial was conducted during 20142016 at Alzahra Teaching Hospital, affiliated with Tabriz University of Medical Sciences, Iran.

Based on the results of previous studies, the determined sample size was at least 48 patients in each group considering $80 \%$ power. Random sampling was used to 
allocate patients to the groups. RandList software (version 2.1, Germany with sequentially numbered containers) was used for randomization and eligible patients were randomly allocated to the intervention groups.

Participants who had abnormal uterine bleeding with disordered proliferative or hyperplastic endometrium with or without atypical status were included in the study. Patients who had allergy to metformin, and not taking medications of bi-guanidinium group (metformin), those who had renal failure, nausea, vomiting, anorexia, anemia, cutaneous lesions, severe hepatic dysfunction, uncontrolled hypertension, thromboembolic disorders, and genital cancers or not satisfied to participate in the study, were excluded. In group 1, metformin $500 \mathrm{mg}$ (Glucophage, Saponine Pharmaceutical CO.), twice a day with megestrol acetate (Gestiran, Iran Hormone CO.), $40 \mathrm{mg}$ daily was prescribed for 3 months and in group 2 megestrol acetate was prescribed $40 \mathrm{mg}$ daily for 3 months. Two weeks after treatment completion, endometrial sampling was performed and the response to treatment was evaluated by comparing the pathologic results.

Early outcome measures were improvement of the symptoms and endometrial histology changes.

In this study, the patients and physician were aware of the type of treatment and the pathologist was blind to the treatment. Patients' demographics, and variables including the number of abortions, pathological findings, hematocrit, and thickness of endometrium were compared.

Sample size estimates were based on the data for the standard deviation of the change in endometrial thickness (mm) from a pilot study. We powered the study to detect a 3-mm difference in the primary end point. To achieve this power, we originally targeted a sample size of 80 randomized individuals. We estimated that we would have $80 \%$ power to detect a $3-\mathrm{mm}$ difference in the primary end point. With twice the dropout rate, our minimum detectable difference for the primary end point would be only slightly higher, at $3.5 \mathrm{~mm}$ between the 2 arms.

Continuous variables were summarized by mean and standard deviation. Demographics and baseline characteristics for participants were summarized by mean and standard deviation for continuous variables and percentages for categorical variables. Normality of variables was examined using Shapiro-Wilk test. In order to compare qualitative variables, chi-square statistical test and Fisher exact test were used and for quantitative variables, independent $t$ test and paired samples' $t$ test were used. SPSS version 18.0 statistical software was used to analyze the data. A $P<0.05$ was considered statistically significant.

\section{Results}

Ninety-six patients were enrolled and included in the study. Eighty-four participants (44 patients in the megestrol acetate plus metformin group and 40 patients in the megestrol acetate group) completed the study. Twelve cases were excluded from the study; 1 case was expired because of unknown cause, 4 cases changed their therapeutic approach, 5 patients had no possibility of following up and 3 cases did not accept re-sampling after the first course of treatment. The consort flow diagram is demonstrated in Figure 1, and the demographic and paraclinical characteristics of 2 groups are depicted in Tables

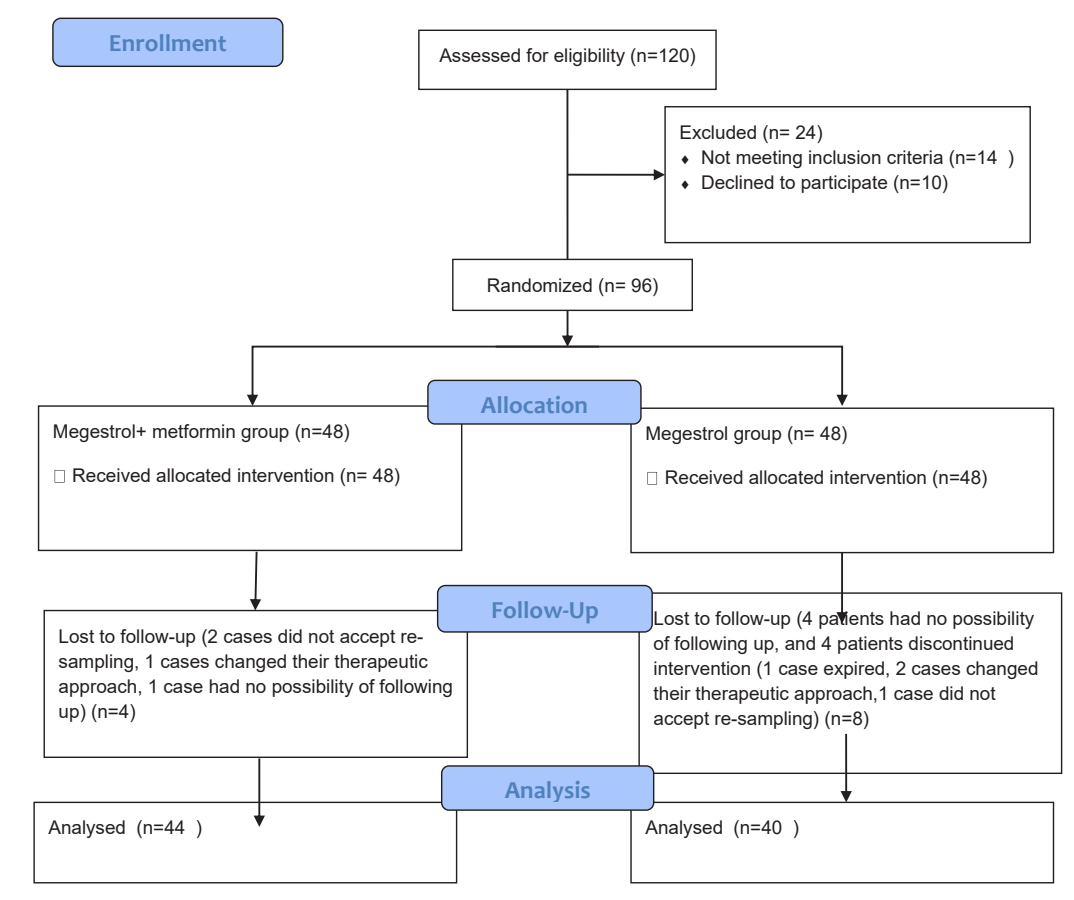

Figure 1. CONSORT Flow Diagram of Patients. 
1 and 2. As shown, there were no significant differences between basic findings of the 2 groups.

Significant differences between groups before and after the intervention were seen only in the thickness of the endometrium $(P<0.001)$. However, in megestrol acetate plus metformin group significant differences was not found in term of changes in endometrial thickness $(P=0.05)$ (Table 2).

Figure 2 demonstrates pathological findings in both groups before the intervention and Figure 3 shows the same findings after the intervention in a comparative method. As shown in Figure 2, for both groups common finding was disordered proliferative endometrium and there was no significant statistical difference between the 2 groups $(P=0.42)$.

According to Figure 2, frequency of endometrial hyperplasia was significantly reduced in both groups. Due to the inappropriate distribution of data, a meaningful evaluation of the distribution of data between the 2 groups was impossible.

With respect to the pathological findings before and after the intervention, 5 cases did not respond to the treatment $(6 \%)$ (2 cases from megestrol acetate group $(4.9 \%)$ and 3 cases $(7 \%)$ from metformin plus megestrol acetate group). It is obvious that treatment refuse rate was lower in the megestrol group, but there was no statistical significant difference between the 2 groups $(P=0.68)$.

\section{Discussion}

Endometrial hyperplasia usually presents with irregular vaginal bleeding and is associated with a long history of unopposed estrogen exposure. Endometrial hyperplasia especially complex atypical hyperplasia is a known precursor of endometrial carcinoma (12). Several treatment regimens have been advocated for treatment. Continuous treatment with megestrol acetate was effective in improving proliferative and hyperplastic disorders of endometrium but its effect on atypical cases was lower (13). Dastranj et al compared the effect of megestrol acetate with metformin and found a higher effect in the metformin group (95.5\%) than the megestrol group (63.6\%) (14). In comparison to this study, we investigated the comparative effect of combination of megestrol acetate and metformin with megestrol acetate alone on proliferative and hyperplastic endometrial disorders. Our results showed appropriate response in both groups without significant differences. However, the response was lower in the metformin group (4.9\% vs $7 \%$ ). In contrast to our study, Gunderson et al reported a $95 \%$ positive response to megestrol acetate (15), which was lower than that in our study (95.1\%).

In a pilot study, Shan et al compared the effect of treatment with combination of megestrol acetate and metformin with megestrol acetate alone on endometrial hyperplasia. Complete response rate was $75 \%$ in metformin group and 25\% in megestrol group (11). Their results contradicted to our study. Their study showed at least one symptom of metabolic syndrome, which could be the cause of the high reported effect. In our study, this possibility was excluded. In this study, we reported more responses in both groups compared to the results of other studies $(95.1 \%$ and $93 \%)$.

Table 1. The Characteristics of the Studied Groups

\begin{tabular}{|c|c|c|c|}
\hline \multirow{2}{*}{ Variables } & \multicolumn{3}{|c|}{ Groups } \\
\hline & Megestrol Acetate+ Metformin (Group 1) $(n=44)^{a}$ & Megestrol acetate (Group 2) $(n=40)^{a}$ & $P$ Value ${ }^{b}$ \\
\hline Age (y) & $46.37 \pm 6.23$ & $43.34 \pm 7.08$ & 0.06 \\
\hline Gravid & $2.96 \pm 1.66$ & $3.21 \pm 1.76$ & 0.48 \\
\hline Para & $2.63 \pm 1.40$ & $2.58 \pm 1.25$ & 0.87 \\
\hline Abortion & $0.35 \pm 0.10$ & $0.58 \pm 0.16$ & 0.24 \\
\hline
\end{tabular}

a Data are presented as mean \pm sandard deviation ). ${ }^{\mathrm{b}} P<0.05$ was considered statistically significant.

Table 2. Paraclinical Findings in 2 Studied Groups

\begin{tabular}{|c|c|c|c|}
\hline \multirow[b]{2}{*}{ Variables } & \multicolumn{2}{|c|}{ Groups } & \multirow{2}{*}{$\begin{array}{l}P \text { Value Between } \\
\text { Group }^{b}\end{array}$} \\
\hline & $\begin{array}{l}\text { Megestrol Acetate+ Metformin } \\
\text { (Group 1) }(n=44)^{a}\end{array}$ & $\begin{array}{l}\text { Megestrol Acetate (Group 2) } \\
\qquad(\mathrm{n}=40)^{a}\end{array}$ & \\
\hline Hematocrit (before hysterectomy) & $36.27 \% \pm 4.17$ & $36.58 \% \pm 4.16$ & \\
\hline Hematocrit (after hysterectomy) & $37.66 \% \pm 3.15$ & $38.62 \% \pm 3.90$ & 0.76 \\
\hline$P$ value within groups ${ }^{b}$ & 0.091 & 0.021 & \\
\hline Endometrial thickness $(\mathrm{mm})$ (before hysterectomy) & $11.97 \pm 7.45$ & $12.73 \pm 6.04$ & \\
\hline$P$ value within group ${ }^{b}$ & 0.056 & $<0.001$ & 0.91 \\
\hline Endometrial thickness (mm) (after hysterectomy) & $8.64 \pm 3.49$ & $8.55 \pm 3.43$ & \\
\hline
\end{tabular}

${ }^{\text {a }}$ Data are presented as mean \pm sandard deviation ). ${ }^{\text {b }} P<0.05$ was considered statistically significant. 


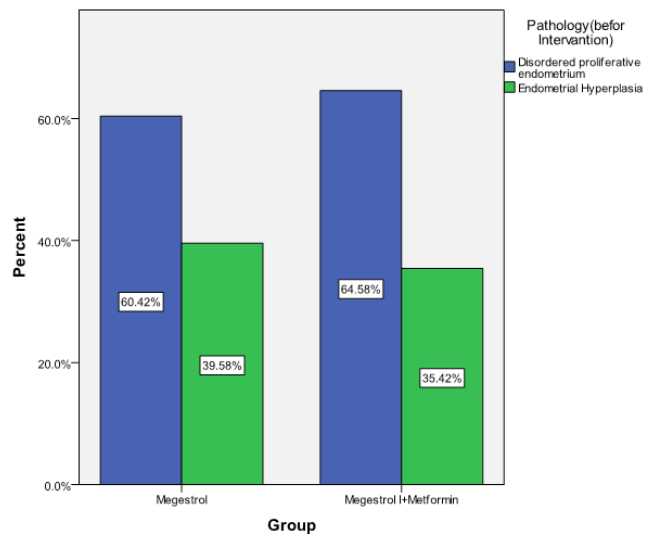

Figure 2. Percentage of Pathological Findings Before Intervention in Both Groups.

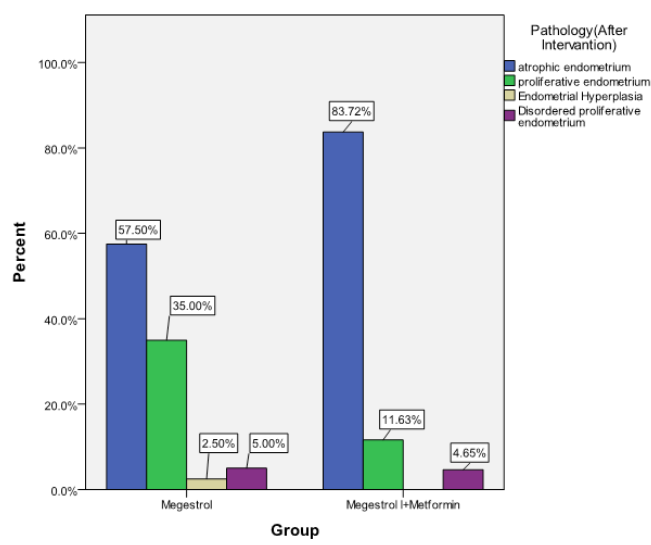

Figure 3. Pathological Findings After Intervention in Both Groups.

As with the study of Shan et al (11) and Dastranj et al (14), we had no complication in any of the groups.

Metformin is an oral antidiabetic agent which is prescribed for type 2 diabetes. In recent years, its effectiveness and safety have been proven in the treatment of cancer $(8,10)$, polycystic ovarian syndrome (16), and some other diseases (17).

In addition to the positive effects of metformin on improving endometrial response to progestin in vivo, it was also shown that metformin has multiple effects. It has recently been identified that metformin has an anticancer effect and is used in combination with chemotherapeutic agents to reduce the dose of chemodrug and increase the efficacy. Metformin works on endometrial cancer through inhibition of cell proliferation and modulation of the mTOR pathway (18) and increases response to treatment (19). Metformin acts through increasing the expression of PgR gene (20), reducing the expression of glyoxalase I (21), regulating rapamycine pathway, an additive effect on AMPK phosphorylation in the ECC-1 cell line, and blocking epidermal growth factor signaling pathway in order to inhibit cellular proliferation and improve progestin treatment (22). Metformin has also been used in several cancers to improve the efficacy of the treatment (23-28). Compared to other studies, it seems that observing more therapeutic results in the progestin group is related to preventing emerging effectiveness of metformin. Therefore, it was clear that adding metformin to megestrol treatment may improve prognosis.

Although, in the present study, different classes of endometrial proliferative disorders were evaluated, performing further investigations with respect only to the patients with endometrial hyperplasia might produce better and the most exact outcomes in this subject.

The limitation to this study was the exclusion of unmarried women because we could not perform endometrial biopsy.

\section{Conclusions}

Both treatment strategies showed effective results. Improvement of pathologic endometrial hyperplasia was seen in most cases of both groups. The treatment methods were tolerated well by the patients and there were no complications. However, in this study adding metformin to megestrol acetate therapy did not increase the effectiveness of treatment.

\section{Ethics Issues}

The Medical Ethics Committee of Tabriz University of Medical Sciences approved the research study. All participants were given adequate information regarding the study, and written informed consent was obtained from the patients. This study was also registered at the Iranian Registry of Clinical Trials (http://www.irct.ir/, identifier: IRCT201410275283N11).

\section{Conflict of Interests}

The authors declare that they have no conflicting of interests.

\section{Financial Support}

This study was funded by a grant from the Women's Reproductive Health Research Center, Tabriz University of Medical Sciences.

\section{Acknowledgements}

We would like to acknowledge all participants who made this study possible.

\section{References}

1. Tran AQ, Gehrig P. Recent Advances in Endometrial Cancer. F1000Res. 2017;6:81. doi:10.12688/f1000research.10020.1

2. Zyla MM, Wilczynski JR, Kostrzewa M, et al. The significance of markers in the diagnosis of endometrial cancer. Prz Menopauzalny. 2016;15(3):176-185. doi:10.5114/pm.2016.63500

3. Anastasiadis PG, Skaphida PG, Koutlaki NG, Galazios GC, Tsikouras PN, Liberis VA. Descriptive epidemiology of endometrial hyperplasia in patients with abnormal uterine bleeding. Eur J Gynaecol Oncol. 2000;21(2):131-134. 
4. Ricciardi E, Maniglio P, Frega A, Marci R, Caserta D, Moscarini M. Fertility-sparing treatment of endometrial cancer precursors among young women: a reproductive point of view. Eur Rev Med Pharmacol Sci. 2012;16(14):1934-1937.

5. Gallos ID, Shehmar M, Thangaratinam S, Papapostolou TK, Coomarasamy A, Gupta JK. Oral progestogens vs levonorgestrel-releasing intrauterine system for endometrial hyperplasia: a systematic review and metaanalysis. Am J Obstet Gynecol. 2010;203(6):547.e541-510. doi:10.1016/j. ajog.2010.07.037

6. Penner KR, Dorigo O, Aoyama C, et al. Predictors of resolution of complex atypical hyperplasia or grade 1 endometrial adenocarcinoma in premenopausal women treated with progestin therapy. Gynecol Oncol. 2012;124(3):542-548. doi:10.1016/j.ygyno.2011.11.004

7. Shen ZQ, Zhu HT, Lin JF. Reverse of progestin-resistant atypical endometrial hyperplasia by metformin and oral contraceptives. Obstet Gynecol. 2008;112(2 Pt 2):465-467. doi:10.1097/AOG.0b013e3181719b92

8. Campagnoli C, Abba C, Ambroggio S, Brucato T, Pasanisi P. Life-style and metformin for the prevention of endometrial pathology in postmenopausal women. Gynecol Endocrinol. 2013;29(2):119-124. doi:10.3109/09513590.2012.706671

9. Session DR, Kalli KR, Tummon IS, Damario MA, Dumesic DA. Treatment of atypical endometrial hyperplasia with an insulin-sensitizing agent. Gynecol Endocrinol. 2003;17(5):405-407.

10. Zhang P, Li H, Tan X, Chen L, Wang S. Association of metformin use with cancer incidence and mortality: a meta-analysis. Cancer Epidemiol. 2013;37(3):207-218. doi:10.1016/j.canep.2012.12.009

11. Shan W, Wang C, Zhang Z, et al. Conservative therapy with metformin plus megestrol acetate for endometrial atypical hyperplasia. J Gynecol Oncol. 2014;25(3):214-220. doi:10.3802/jgo.2014.25.3.214

12. Barakat RR, Markman M, Randall M. Principles and practice of gynecologic oncology. Philadelphia: Lippincott Williams Wilkins; 2009.

13. Novak E, Berek JS. Berek and Novak's Gynecology. Philadelphia: Lippincott Williams Wilkins; 2007.

14. Tabrizi AD, Melli MS, Foroughi M, Ghojazadeh M, Bidadi S. Antiproliferative effect of metformin on the endometrium--a clinical trial. Asian Pac J Cancer Prev. 2014;15(23):10067-10070.

15. Gunderson CC, Dutta S, Fader AN, et al. Pathologic features associated with resolution of complex atypical hyperplasia and grade 1 endometrial adenocarcinoma after progestin therapy. Gynecol Oncol. 2014;132(1):33-37. doi:10.1016/j. ygyno.2013.11.033

16. Ganie MA, Khurana ML, Nisar S, et al. Improved efficacy of low-dose spironolactone and metformin combination than either drug alone in the management of women with polycystic ovary syndrome (PCOS): a six-month, open-label randomized study. J Clin Endocrinol Metab. 2013;98(9):3599-3607. doi:10.1210/jc.2013-1040
17. Lexis CP, van der Horst IC, Lipsic E, et al. Metformin in nondiabetic patients presenting with ST elevation myocardial infarction: rationale and design of the glycometabolic intervention as adjunct to primary percutaneous intervention in ST elevation myocardial infarction (GIPS)III trial. Cardiovasc Drugs Ther. 2012;26(5):417-426. doi:10.1007/s10557-012-6413-1

18. Zhang HH, Guo XL. Combinational strategies of metformin and chemotherapy in cancers. Cancer Chemother Pharmacol. 2016;78(1):13-26. doi:10.1007/s00280-0163037-3

19. Peng $M$, Darko KO, Tao $T$, et al. Combination of metformin with chemotherapeutic drugs via different molecular mechanisms. Cancer Treat Rev. 2017;54:24-33. doi:10.1016/j.ctrv.2017.01.005

20. Xie Y, Wang YL, Yu L, et al. Metformin promotes progesterone receptor expression via inhibition of mammalian target of rapamycin (mTOR) in endometrial cancer cells. J Steroid Biochem Mol Biol. 2011;126(35):113-120. doi:10.1016/j.jsbmb.2010.12.006

21. Zhang Z, Dong L, Sui L, et al. Metformin reverses progestin resistance in endometrial cancer cells by downregulating GloI expression. Int J Gynecol Cancer. 2011;21(2):213-221. doi:10.1097/IGC.0b013e318207dac7

22. Hanna RK, Zhou C, Malloy KM, et al. Metformin potentiates the effects of paclitaxel in endometrial cancer cells through inhibition of cell proliferation and modulation of the mTOR pathway. Gynecol Oncol. 2012;125(2):458469. doi:10.1016/j.ygyno.2012.01.009

23. Kim HJ, Kwon H, Lee JW, et al. Metformin increases survival in hormone receptor-positive, HER2-positive breast cancer patients with diabetes. Breast Cancer Res. 2015;17:64. doi:10.1186/s13058-015-0574-3

24. Kong F, Gao F, Liu H, et al. Metformin use improves the survival of diabetic combined small-cell lung cancer patients. Tumour Biol. 2015;36(10):8101-8106. doi:10.1007/ s13277-015-3549-1

25. Zhang Y, Guan M, Zheng Z, Zhang Q, Gao F, Xue Y. Effects of metformin on CD133+ colorectal cancer cells in diabetic patients. PLoS One. 2013;8(11):e81264. doi:10.1371/ journal.pone.0081264

26. Donadon V, Balbi M, Mas MD, Casarin P, Zanette G. Metformin and reduced risk of hepatocellular carcinoma in diabetic patients with chronic liver disease. Liver Int. 2010;30(5):750-758. doi:10.1111/j.1478-3231.2010.02223.x

27. Kowall B, Stang A, Rathmann W, Kostev K. No reduced risk of overall, colorectal, lung, breast, and prostate cancer with metformin therapy in diabetic patients: database analyses from Germany and the UK. Pharmacoepidemiol Drug Saf. 2015;24(8):865-874. doi:10.1002/pds.3823

28. Chen G, Xu S, Renko K, Derwahl M. Metformin inhibits growth of thyroid carcinoma cells, suppresses self-renewal of derived cancer stem cells, and potentiates the effect of chemotherapeutic agents. J Clin Endocrinol Metab. 2012;97(4):E510-520. doi:10.1210/jc.2011-1754

(c) 2018 The Author (s); This is an open-access article distributed under the terms of the Creative Commons Attribution License (http://creativecommons.org/licenses/by/4.0), which permits unrestricted use, distribution, and reproduction in any medium, provided the original work is properly cited. 